\title{
A Research on the Recent Advances in the Development of Energy Efficient Distributed Cooperative Routing Protocol for Wireless Sensor Networks in the Current Scenario
}

\author{
Raghavendra Patil G.E., Thippeswamy M.N.
}

\begin{abstract}
A brief exhaustive summary review of the various advances in the development of energy efficient distributed cooperative routing protocol for wireless sensor $n / w$ 's in the current scenario is presented in a nut shell in this research article. This paper just gives a in-depth scenario into the various works of the authors \& lays a foundation for the proposition of the works that could be done by the future researchers in the area of wireless sensor networks.
\end{abstract}

Keywords : Cooperative Routing, Energy Efficiency, Packet Delivery Ratio, Throughput, Channel Quality Indicator, Wireless Sensor Networks (WSNs).

\section{INTRODUCTION}

In remote Sensor Networks, with the upside of communicate in remote medium, the Cooperative correspondence has turned out to be increasingly more alluring as of late. Agreeable correspondence which permits a few hubs helpfully transmit sign to a goal together. In helpful frameworks, a gathering of hubs can transmit all together reception apparatus exhibit to get decent variety gains. Collaboration can fundamentally improve the exhibition of remote systems; agreeable directing can give expanded transmission extend, improved vitality and transfer speed proficiency, and increasingly dependable and longer enduring system network.

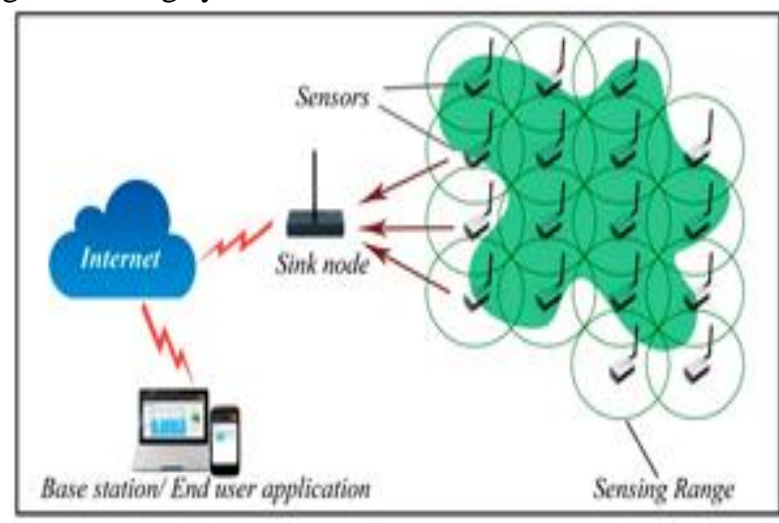

Fig. 1 : A typical WSN-1
However, in large, complex, dynamic networks. Centralized control will probably be infeasible, and the overhead required for communications between the cooperating nodes could be excessive. To address these issues, developing and analysing distributed cooperative strategies which work well without concentrated control or full between hub interchanges, and which are put together however much as could reasonably be expected with respect to privately acquired data. A pictorial perspective on the WSN is appeared in the figure.

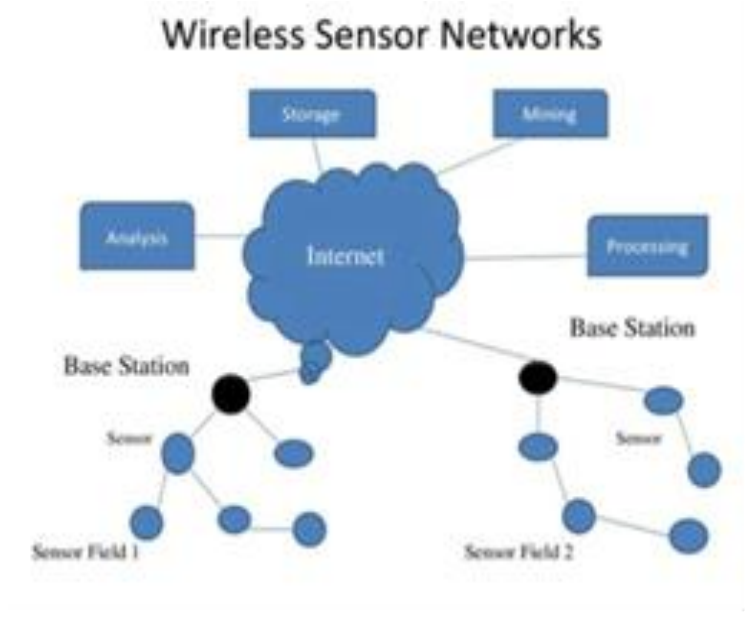

Fig. 2 : A typical WSN-2

\section{LITERATURE SURVEY}

In the modern day technological world, automation plays a very important role in the human life ranging from domestic applications to the industrial applications. This automation makes use of various technological devices such as machines, computers \& its accessories, etc... which could be used by the humans for various applications. Hence, networking plays a very important role. Each component can be treated as a wireless sensor network. A brief insight into the same was presented in the introductory chapter. In this chapter, a brief overview of the work done in the relevant field was conducted on the chosen research topic "An energy efficient distributed cooperative routing protocol for wireless sensor networks" and the same was presented in the form of an exhaustive literature survey [1] - [60], which was the content of our another research paper.

Revised Manuscript Received on 14 August, 2019.

Raghavendra Patil G.E., Research Scholar, VTU Research Centre, NitteMeenakshi Institute of Technology, NMIT, Bangalore \& Asst. Prof., CSE Dept., SDMIT, Shree DharmasthalaManjunatheswara Institute of Technology, Dharmasthala Road, Near Siddhavana, Ujire, Karnataka, South Kanara, Karnataka, India. (Email : gerpatill16@ @gmail.com)

Dr. Thippeswamy M.N., Professor \& Head, Computer Science \&Engg., NitteMeenakshi Institute of Technology, NMIT, Yelahanka, Bangalore, Karnataka, India.. 
Various specialists/creators have chipped away at the control of remote sensor systems till date in different limits and in all round angles, beginning from displaying, examination, recreationupto the usage level. To start with, 100s of research papers were collected from various sources, studied @ length \& breadth and a review paper was published by us to start with in the field of the control aspects related to the research work undertaken.

Wireless Sensor networks consists of small, low-power, and low-cost devices with limited computational and wireless communication capabilities. They represent the next step in wireless communication's miniaturization, and their power and size make it feasible to embed them into wearable vital sign monitors, location-tracking tags in buildings, and first responder uniform gear. A major problem for sensor networks applications is to determine the efficient way of conserving the energy as nodes have extreme resource limitations [26]. Cooperative diversity and communications techniques are well known for mitigating the effects of multipath fading and improving the reliability of communication in wireless channels [27]. A new class of schemes called cooperative communication has been developed recently which enables the nodes with a singleantenna in a multiuse environment to share their antennas and generate a virtual multiple-antenna transmitter that allows them to achieve transmit diversity [28, 29].

In [24], the authors proposed a novel location-based cooperative routing scheme for WSNs that takes advantage of the low link power and high channel gain of the cooperative routing WSNs. The proposed routing algorithm also works well in the case where the transmission power is low. The proposed protocol in [24] uses the RTS/CTS handshaking mechanism to find the node's location information. The principle thought of the calculation in [24] is that every hub utilizes it's transmit control furthest limit as it's transmit control so as to guarantee enough transmission separation if there should be an occurrence of low vitality. In [24], the transmission separation and the blackout likelihood, can be determined utilizing the most minimal connection control, will commonly impact one another, and after that the sending hub will utilize the determined transmission separation as the reason for choosing the area of the following bounce hub.

\section{RESULTS \& DISCUSSIONS}

A dispersed vigorous steering convention is proposed in [25] which upgrade the power of directing against way breakage by enabling hubs to work helpfully. In [25], the protocol selects the path which can be adapted to the varying topology based on the path quality and it also uses path diversity to select the best path for each packet. The problem with this protocol is usage of control packets while selecting the suitable path for the packet. A new energy efficient cooperative routing scheme to improve the link's energy efficiency using space-time block codes is proposed in [30]. In [30], the selected multiple nodes act as multiple transmitting and receiving antennas. In [31], the authors analysed the system performance from the network aspect. The work used an M/G/1 queuing model [32] to analyse the delay and throughout performance of the system. The performance was then studied using the Pollaczek-
Khintchine (P-K) formula [33] and the steady state distribution of an embedded Markov chain model.

The work done in [34] proposed a novel cooperative contention-based forwarding protocol for geographic routing in WSNs. This protocol uses the forwarding opportunities among nearest nodes to improve energy efficiency. The main problem with this protocol is that it uses CTS/RTS control packets exchange to select the next hop nodes in the path. In [35], an energy efficient adaptive cooperative routing that maximizes the network lifetime by reducing energy consumption is proposed. A logical model is created to locate the best area of the sink hub to lessen the separation between the sources and the sink. The calculation proposed in [35], distinguishes the most limited way with increasingly signal quality for the transmission of information. In this work [36], a directing measurement is inferred that catches the identification blunder prompted by each connection, in a sensible situation. A helpful steering plan, which empowers critical vitality preservation contrasted with regular Minimum-Energy directing and non agreeable directing plans for a given location dependability level for WSNs is proposed.

In this work [37], the creators present a novel plan of a helpful hubs learning plan for agreeable vitality productive directing (CEERA) for remote sensor systems. In CEERA, hubs play out an agreeable learning in conveying information to the base station. The retransmission of bundles is controlled through a location based clock. CEERA accomplishes overhead decrease and vitality protection by controlling different parameters that influence the general system effectiveness. The recreation results appeared in [37] demonstrates that our calculation limits the general vitality utilization of the WSN. The work done in [38], proposes an agreeable directing in versatile specially appointed remote sensor systems with Rayleigh blurring situations. The work in [38] considers the development of stable directing course by utilizing entropy ideas in the view purposes of versatility of hubs. At that point, the agreeable helped transmission utilizing the steady transfer which can be chosen by utilizing entropy ideas is considered. A hypothetical investigation model is proposed for the proposed agreeable directing with the blackout likelihood of the start to finish execution.

In [39], a vitality effective helpful geographic directing (ECGR) to arrive at vitality proficient steering in WSN is proposed. ECGR completely exploits agreeable assorted variety and geographic directing. At physical layer, the sensor hubs helpfully transmit a similar bundle to accomplish more distant transmission separation of each jump. While at the system layer, ECGR adaptively chooses the fitting agreeable hubs to shape sending groups to advance the parcel. So as to completely evaluate the vitality utilization, the creators take circuit vitality utilization of the sensor hubs into thought. The work demonstrates the general system vitality utilization is diminished and adjusted among hubs to broaden the life of the system. In [40], a system coding-based probabilistic directing (NCPR) plot is 
proposed, which is vitality proficient, solid and reduces the communicate storm issue in a grouped WSN. The principle focal point of this work [40] is to plan the system coding-based methodology for probabilistic directing conventions. In NCPR, a sensor hub instates a transmission procedure in a WSN group by transmitting a bundle from its detected line.

Each neighbor hub encodes its got parcel with its detected bundle utilizing XOR system coding and transmits the coded bundle with certain likelihood. Three system codingbased methods have been proposed in [40] for encoding and translating of bundles in intra-bunch and between group interchanges by conveying jobs among the sensor hubs. It has been appeared in [40] that the NCPR plan gives better vitality effectiveness and unwavering quality contrasted with the probabilistic steering and unadulterated flooding plans. In this work [41], the creators proposed a steering convention dependent on traffic grouping and job task to empower heterogeneous WSN for participation. This methodology considers both QoS necessities and lifetime expansion to permit the conjunction of various applications in the heterogeneous system framework. In [42] Cooperative correspondence (CC) enables numerous hubs to at the same time transmit a similar bundle to the collector with the goal that the consolidated sign at the beneficiary can be effectively decoded. Since the $\mathrm{CC}$ can diminish the transmission control and expand the transmission inclusion, it has been considered in least vitality directing conventions to lessen the all out vitality utilization.

The Cooperative correspondence plans to think about the effect of agreeable steering on adjusting the vitality appropriation among hubs. By presenting another directing plan which cautiously chooses helpful transfer hubs and appoints their transmission control, our agreeable steering strategy can adjust the rest of the vitality among neighboring hubs to expand the lifetime of the system. The tale steering plan vitality adjusted agreeable directing (EBCR) [42] to choose helpful hand-off hubs and their transmission control for each bounce. It very well may be applied to any hidden vitality mindful steering convention, and just need neighborhood data to play out the improvement on CCs. We officially demonstrate that our agreeable steering strategy EBCR can for sure balance the vitality among hubs and draw out the rest of the lifetime of the system. Despite the fact that this strategy can adjust the vitality over any vitality mindful steering conventions, it has a few restrictions as well: (1) our proposed plan doesn't work for versatile systems, since hub development will break the ideal determination of agreeable transfers; (2) we accept a disentangled CC model where blurring impact isn't considered.

As of late, the benefits of helpful correspondence in the physical layer have been investigated. Be that as it may, the effect of helpful correspondence on the structure of the higher layers has not been surely known at this point. Agreeable directing in remote systems has increased much enthusiasm because of its capacity to abuse the communicate idea of the remote medium in structuring power effective steering calculations. A large portion of the current collaboration based steering calculations are actualized by finding a most limited way course first and after that improving the course utilizing helpful correspondence. All things considered, these steering calculations don't completely abuse the benefits of helpful correspondences, since the ideal agreeable course probably won't be like the most limited way course. In [43] propose a participation based directing calculation, to be specific, the Minimum Power Cooperative Routing (MPCR) calculation, which utilizes the helpful correspondences while building the base power course.

In [43] The MPCR calculation develops the base power course, which ensures certain throughput, as a course of the base power single-transfer building hinders from the source to the goal. In this way, any disseminated most limited way calculation can be used to locate the ideal helpful course with polynomial intricacy. Proposed Routing Algorithms First, a participation based steering calculation, specifically, the Minimum-Power Cooperative Routing (MPCR) calculation. The MPCR calculation mulls over the agreeable interchanges while developing the base power course. Second, participation based steering calculation, in particular, Cooperation along the Shortest Non-Cooperative Path (CASNCP) calculation. The CASNCP calculation is like the heuristic calculations.

In [44] Efficient power allotment techniques for a decentralized appropriated space-time square coding (DisSTBC) framework where learning about the channel state data (CSI) isn't accessible at the transmitter(s). The first is an open-circle procedure which requires no control flagging; the second is a criticism helped methodology which requires some control flagging, yet which can accomplish better control proficiency. [44] Focusing on a specific decentralized Dis-STBC conspire. In [45] helpful transmission, each hub on the way from the source hub to the goal hub turns into a bunch head, with the undertaking of selecting different hubs in its neighbourhood and organizing their transmissions. Subsequently, the old-style course from a source hub to a sink hub is supplanted with a multihop agreeable way, and the old-style point-to-point correspondence is supplanted with many-to-numerous helpful correspondence.

The agreeable transmission convention comprises of two stages. In the steering stage, the underlying way between the source and the sink hubs is found as a fundamental "one-hub thick" way. At that point, the way experiences a thickening procedure in the "enlisting and-transmitting" stage. In this stage, the hubs on the underlying way become bunch heads, which enlist extra nearby hubs from their neighbourhood. It has been appeared in [42, 44] Cooperative transmission convention with another helpful convention, called Cooperation Along Non-agreeable way (CAN) and with two other non-helpful plans: the "disjoint-ways" and "one-way" plans. The absolute vitality utilization was scientifically figured, delineating significant vitality reserve funds. For instance, when hubs are situated on a network, the vitality investment funds of proposed helpful convention over the CAN convention is up to $80 \%$. 


\section{A Research on the Recent Advances in the Development of Energy Efficient Distributed Cooperative Routing Protocol for Wireless Sensor Networks in the Current scenario}

Presenting a helpful correspondence convention for agreeably transmitting information and association of groups. In [45] the proposed framework diminishes the vitality utilization dependent on the correspondence model, which considers just the correspondence however not the parameters of the hubs in the agreeable systems. Further vitality can be limited by utilizing an enhancement system to limit the general vitality utilization. The complete vitality usage can be progressively decreased by altering the transmit control for intra-bunch and between group transmission. The Cooperative transmission convention comprises of two stages the stages are directing stage and enrolling and transmitting. The steering period of the convention, which is in charge of finding a "one-hub thick" course from the source hub to the sink hub, should be possible utilizing one of the numerous recently distributed directing conventions. In [44] For the motivation behind execution assessment, Author proposed to actualize this stage utilizing the Ad hoc On-request Distance-Vector directing convention (AODV) with certain alterations and with the connections transmissions vitality utilized as the connections cost. The "enlisting and-transmitting" stage-is done powerfully per bounce, beginning from the source hub and proceeding onward, jump by jump, as the parcel moves along the way to the sink hub. To improve the further vitality we are utilizing streamlining method in particular subterranean insect state advancement calculation.

In [45] helpful remote correspondence, we are worried about remote sensor hubs, which may expand their productivity in nature of administration by methods for participation. In a helpful correspondence framework, every remote sensor hubs can transmit the information just as go about as an agreeable operator for another remote sensor hub. In agreeable correspondence every client transmits their information with the goal that information misfortune can be decreased. Then again, the proficiency of every client will be improved in light of the fact that, of the collaboration assorted variety, the channel code rates can be expanded. The fundamental inquiry, regardless of whether participation is worth to be actualized, has been addressed emphatically. In agreeable correspondence, data will be shared among a couple of hubs and communicate commonly as a virtual receiving wire cluster.

In sensor systems vitality is the principle imperative, [45] present looks into are going on and consideration concentrated on the vitality effectiveness of the agreeable correspondence and expectation that it will spare vitality and broaden the systems lifetime. It is alluded that helpful correspondence in a run of the mill group based remote sensor organize that spares vitality productively. Likewise it is demonstrated that notwithstanding when both the transmission vitality and the circuit vitality utilization are considered, helpful correspondence can in any case significantly lessen the complete vitality utilization. So we put a helpful correspondence plot into a multi-bounce remote system to lessen both vitality utilization and postponement. Despite the fact that agreeable correspondence is vitality proficient, very little work has been done on the best way to accomplish this in the vitality obliged systems. In the steering period of the convention, which is in charge of finding a "one-hub thick" course from the source hub to the sink hub, could be actualized utilizing one of the numerous recently distributed directing conventions. With the end goal of execution assessment, in [44, 45$]$ Author actualized this stage utilizing the Ad hoc On-request Distance-Vector directing convention (AODV) and with the connections' transmissions vitality utilized as the connections' expense.

In [46] Cooperative correspondence in remote systems has turned out to be increasingly more appealing as of late since it could moderate the especially serious channel hindrances emerging from multipath proliferation. Here the more prominent advantages picked up by abusing spatial assorted variety in the channel. Cross-layer plan for remote sensor systems to improve the system execution. The [46] paper shows another vitality proficient helpful directing plan with space decent variety utilizing space-time square codes (STBCs) simultaneously as improving the connection's vitality effectiveness. In our answer, the chose numerous hubs go about as different transmitting and getting recieving wires. Full decent variety from the symmetrical STBC is used to defeat multipath blurring and to improve control effectiveness. The system execution measures, for example, organize throughput and postponement are broke down by means of a M/G/1 lining model [9]. The vitality utilization and convention proficiency investigated.

The creator proposed in [47] Wireless systems transmit data from a source to a goal by means of numerous jumps so as to spare vitality and, in this manner, increment the lifetime of battery-worked hubs. The vitality investment funds can be particularly huge in agreeable transmission plans, where a few hubs coordinate during one jump to advance the data to the following hub along a course to the goal. In [47] finding the best multi-bounce transmission strategy in such a system which decides hubs that are associated with each jump, is a significant issue. The issue of processing a vitality ideal agreeable transmission conspire in a remote system for two distinctive channel blurring models: I moderate blurring channels, where the channel additions of the connections continue as before for countless transmissions, and ii quick blurring channels, where the channel increases of the connections change rapidly starting with one transmission then onto the next. For moderate blurring, we think about a considered class of approaches (comparing to neighborhood participation among hubs), and demonstrate that the calculation of an ideal arrangement in this class is proportionate to a briefest way calculation on a prompted diagram, whose edge expenses can be registered in a decentralized way utilizing just locally accessible channel state data (CSI). For quick blurring, both CSI procurement and information transmission expend vitality.

In [48] the circulated nature and dynamic topology of Wireless Sensor Networks (WSNs) presents unique necessities in steering conventions that ought to be met. The most significant component of a steering convention, so as to be effective for WSNs, is the vitality utilization and the expansion of the system's lifetime. WSN is a gathering of remote hubs with restricted vitality capacities that might be

Blue Eyes Intelligence Engineering

\& Sciences Publication 
portable or stationary and are found haphazardly on a progressively evolving condition. The directing systems choice is a significant issue for the effective conveyance of the parcels to their goal. Besides, in such organizes, the applied directing procedure ought to guarantee the base of the vitality utilization and consequently boost of the lifetime of the system. In [48] the fundamental waste of battery is because of transmitting and accepting information among hubs and the handling components.

- $\quad$ Average Energy Dissipated: This measurement is identified with the system lifetime and demonstrates the normal dispersal of vitality per hub after some time in the system as it performs different capacities, for example, transmitting, accepting, detecting and collection of information.

- Low Energy Consumption: A low vitality convention needs to devour less vitality than customary conventions. This implies a convention that thinks about the rest of the vitality level of the hubs and chooses courses that expand the system's lifetime is considered as low vitality convention.

- Total Number of Nodes Alive: This measurement is additionally identified with the system lifetime. It gives a thought of the zone inclusion of the system after some time.

- Total Number of Data Signals Received at BS: This measurement is comparable to the vitality spared by the convention by not transmitting ceaselessly information

- Average Packet Delay. This measurement is determined as the normal single direction idleness that is seen between the transmission and gathering of an information bundle at the sink. This measurement estimates the fleeting exactness of a parcel.

- Packet Delivery Ratio. It is determined as the proportion of the quantity of particular parcels got at sinks to the number initially sent from source sensors. This measurement shows the unwavering quality of information conveyance.

- Time until the First Node Dies: This measurement shows the span for which all the sensor hubs on the system are alive. There are conventions in which the principal hub on the system comes up short on vitality sooner than in different conventions, however figures out how to keep the system operational any longer.

- Energy Spent per Round: This measurement is identified with the aggregate sum of vitality spent in directing messages in a round. It is a transient measure intended to give a thought of the vitality proficiency of any proposed technique in a specific round.

- Idle Listening: A sensor hub that is out of gear listening mode, doesn't send or get information, it can in any case devour a significant measure of vitality. Along these lines, this hub ought not remain out of gear listening mode, yet ought to be controlled off.

- $\quad$ Packet Size: The size of a parcel decides the time that a transmission will last. Hence, it is successful in vitality utilization. The parcel size must be diminished by joining a few bundles into one huge bundle or by Compression. bundles (hi messages), which are not required.

- Distance: The separation between the transmitter and collector can influence the power that is required to send and get parcels. The directing conventions can choose the briefest ways among hubs and decrease vitality utilization.

In [49] the creator proposed the Distributed Cooperative Routing and Learning. the circulated strategy to execute the greatest lifetime agreeable steering calculation. The strategy depends on the circulated fortification picking up steering calculation. The directing choice is found out by all hubs in the system. Every hub keeps up the best bundle conveyance cost to every one of the goals. Specifically, every hub I keeps up a table of Q-values Qi $(j, d)$, for $j$ ? Si, where $\mathrm{j}$ is in the arrangement of hub I neighbour'si, $\mathrm{Si}$, and hub $\mathrm{d}$ is the goal. The Qi (j, d) has the elucidation of hub I's best assessed cost that a parcel would bring about to arrive at its goal hub $\mathrm{d}$ from hub I when the bundle is sent by means of hub I's neighbor hub $\mathrm{j}$. The incentive in the Q-table will be traded between hub I and $\mathrm{j}$, at whatever point there is a parcel is sent from hub I and $\mathrm{j}$, and the other way around.

In [50] the organization of remote sensor arranges in numerous application territories like condition control, target following in war zones, requires a streamlining to the correspondence among the sensors in order to serve information in short dormancy and with insignificant vitality utilization. Most of utilizations dependent on remote sensor systems depends predominantly on the capacity of the hidden conventions to scale to huge number of sensors, to moderate vitality and give answers in short inertness. In [50] the creator proposed the business related to the Cooperative information storing has been utilized as a successful and effective strategy to accomplish these objectives simultaneously. The plan of these conventions depends primarily on the determination of the sensor hubs which will take exceptional jobs in running the storing and solicitation sending choices. The article presented another centrality metric that guide to choose such hubs, and proposed another vitality proficient helpful reserving convention.

The ongoing expanded enthusiasm for circulated and adaptable remote inescapable applications has attracted extraordinary consideration regarding Wireless Networked Control Systems (WNCS) structures dependent on Wireless Sensor and Actuator Networks (WSANs) and the subsequent Quality of Service (QoS) acquired in explicit applications. In remote checking frameworks dependent on WSANs, giving certain QoS determinations as far as dependability and vitality productivity is urgent for the sensors/actuators as they perform activities dependent on the information tests/got with a restricted measure of vitality to spend. In [51] presenting the new helpful based steeringcalculation to ensure a decent exhibition exchange off among dependability and vitality proficiency of the general remote observing framework. Reproductions have been done so as to evaluate the effect of the proposed calculation on the general observing framework dependability and vitality productivity and a correlation is given the current Ad-hoc On-Distance Vector (AODV), the 
collaboration along the most limited non-agreeable way (CASNCP) and least control helpful directing (MPCR) calculations

The wide usage of Wireless Sensor Networks (WSNs) is blocked by the seriously restricted vitality limitations of the individual sensor hubs. This is the motivation behind why an enormous piece of the examination in WSNs centers around the improvement of vitality productive steering conventions. In [52] another convention called Equalized Cluster Head Election Routing Protocol (ECHERP), which seeks after vitality protection through adjusted bunching, is proposed. ECHERP models the system as a straight framework and, utilizing the Gaussian end calculation, computes the mixes of hubs that can be picked as group heads so as to expand the system lifetime. In [52] LEACH is a convention that will in general decrease vitality utilization in a WSN. Be that as it may, LEACH uses single-bounce steering in which every sensor hub transmits data straightforwardly to the bunch head or the sink. In this way, it isn't suggested for systems that are conveyed in enormous districts.

Power-Efficient Gathering in Sensor Information Systems (PEGASIS) is an imperativeness beneficial show [53], which gives improvements over LEACH. In PEGASIS, each center point talks about just with a near to neighbor in order to exchange data. It interchanges in order to transmit the information to the base station, along these lines diminishing the proportion of essentialness spent per round. The center points are dealt with in order to outline a chain, which can either be formed by the sensor centers themselves using a covetous estimation starting from a particular center point, or the BS can process this chain and impart it to all the sensor center points. In $\mathrm{LEACH}$, a center point transforms into a gathering head using a stochastic segment. This is slanted to conveying uneven essentialness level holds in center points and, along these lines, to extending the hard and fast imperativeness dispersed in the framework. In PEGASIS, the gathering head decision doesn't consider neither the remaining imperativeness of the center points or the territory of the base station. PEGASIS has better execution appeared differently in relation to LEACH [54], yet the center points are assembled into chains that reason monotonous data transmissions.

Edge Sensitive Energy Efficient (TEEN) is a different leveled show expected for sudden changes in the distinguished condition [55]. The response of the framework in time-fundamental applications is basic, obliging the framework to work in a responsive mode. The sensor arrange plan in TEEN relies upon different leveled assembling. The center points close to upper level bundles are used to move data from various centers that are further away, a method that goes on the accompanying level gathering until the sink is come to. The central favored situation of TEEN is that it capacities honorably in conditions where surprising changes in the distinguished properties occur. On the other hand, in huge district frameworks and when the amount of layers in the hierarchy of leadership is pretty much nothing, TEEN will as a rule eat up broad proportions of essentialness, because of long evacuate transmissions. What's more, when the amount of layers assembles, the transmissions become shorter and there exists a broad overhead in the plan organize, similarly as the movement of the framework.

The Shortest Hop Routing Tree convention (SHORT) [56] productively gathers helpful information from a remote sensor system to the base station and gives vitality effectiveness. This convention chooses the hub with the biggest estimation of remaining vitality as the pioneer. The Extending Lifetime of Cluster Head (ELCH) steering convention [57] has self-setup and hierarchal directing properties. It chooses bunch heads dependent on the votes that it gathers from the system hubs. The Energy Efficient Cluster Formation Protocol (EECFP) [58] chooses the hubs with the higher vitality as bunch heads and pivots them in each round to give a parity of vitality utilization and to limit the vitality spend for group arrangement. In [59], a brought together steering convention, called Base-Station Controlled Dynamic Clustering Protocol (BCDCP), which disperses the vitality scattering uniformly among all the sensor hubs to improve the system lifetime, and its normal vitality reserve funds are displayed. The base station gets the leftover vitality of every hub, and afterward, it figures the normal vitality level of the considerable number of hubs. At that point, it chooses as applicant bunch heads various hubs, which have a higher leftover vitality than this worth. This convention gives a reasonable vitality utilization. Be that as it may, the choice of the hub with the most elevated vitality as a bunch head at a round may make different hubs spend more vitality to send information to this hub. The determination of a hub that permits different hubs in the bunch to spend less vitality is a superior arrangement.

Like this, an enormous number of specialists had dealt with the remote system frameworks and truth be told, just the significant works have been displayed in this writing study. In dominant part of the work done by the various specialists/creators displayed in the past sections, there were parcel of detriments/loads/lacunas/disadvantages/lacks. Countless articles, papers, proposition, reports done by different writers, scientists, engineers, understudies, resources were surfed upon concentrated in a word. Maybe a couple of the downsides [1] - [60] of the works that were done by the previous specialists were considered in our exploration work, contemplated in a word and calculations were created so as to defeat a portion of the insufficiencies of the current algos. The exploration work is checked through successful reenactment brings about the MatlabSimulink condition or in the NS-2 test system stage so as to substantiate the examination issue attempted in correlation with the work done by the previous creators in the important field, in the sense to understand the ideal target (question) and touch base at the result (answer/arrangement) of the examination work.

\section{CONCLUSION AND FUTURE WORK}

In the literature work, existing techniques cooperative communication in wireless Sensor Networks for Energy efficiency are noted and discussed. The work done in the literature has not considered using distributed cooperative

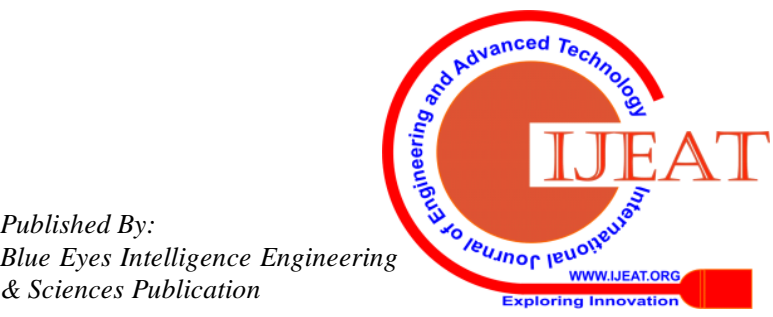


communication to reduce energy consumption in WSNs. The various challenges which are still open for research in the field of Energy efficient cooperative communication for WSNs \&is going to be carried out \& published in our next research articles as contributory works.

\section{REFERENCES}

1. Perkins, E. Belding-Royer, and S. Das, "Ad hoc on-demand distance vector (aodv) routing," United States, 2003

2. Liang, X., Chen, M., Xiao, Y, and Balasingham, (2010). MRL-CC: a novel cooperative communication protocol for QoS provisioning in wireless sensor networks. International Journal of Sensor Networks, 8(2), 98-108.

3. Maria Sefuba, Tom Walingo, "Energy-efficient medium access IET Wirel. Sens. Syst., 2018, Vol. 8 Iss. 3, pp. 99-108.

4. Ibriq, J., Mahgoub, I.: 'Cluster-based routing in wireless sensor networks: issues and challenges'. SPECTS, 2004, pp. 759-766

5. Shan. 1.. Dong. L.. Liao. X., Shao. L.. Gao. Z .. and Gao. Y(2013). Research on improved Leach Protocol of wireless sensor networks PRZEGLD ELEKTROTECHNICZNY, ISSN, 0033-2097

6. AI-Rahayfeh, A. A., and Almiani, M. M. (2014). Parameterized Affect of Transmission-Range on Lost of Network Connectivity (LNC) of Wireless Sensor Networks.

7. Hussain, M and Mottalib. M. (2011). Energy-Efficient Hierarchical Routing Protocol for Homogeneous Wireless Sensor Network. I]CSNS International Journal of Computer Science and Network Security, 1 (I), 80-86

8. Huang. X Zhai. H .. and Fang. Y (2008). Robust cooperative routing Communications, IEEE Transactions on, 7(12), 5278-5285.

9. Wang, J.; Kim, ].-U.; Shu, L.; Niu, Y; Lee, S. A distance-based energy aware routing algorithm for wireless sensor networks. Sensors 2010. 10. 94939511.

10. Wail Mardini; Yaser Khamayseh; Shorouq AL-Eide. Optimal Number of Relays in Cooperative Communication in Wireless Sensor Networks, Communications and Network Journal in Vol. 4, No. 2 Issue (May 2012). ISSN 1949-2421. USA.

11. Raghavendra Patil G E, Dr. Thippeswamy M N "The Energy Efficien Dynamic Source Routing Protocol For Manet" Proceedings of 2nd Inter National Conference on Networks Information and Communication, 2015 (ICNIC-2015), Sri Venkateshwara College of Engineering, Bangalore, India. May18th-20th 2015.

12. A.F. M. Shahen Shah, Md. Shariful Islam, " A Survey on Cooperative Communication in Wireless Networks" I.J. Intelligent Systems and Applications, 2014, 07, 66-78 Published Online June 2014 in MECS (http://www.mecs-press.org/) DOI 10.5815/ijisa.2014.07.09,

13. D. Zhang, G. Li, K. Zheng, X. Ming, and Z. H. Pan, "An EnergyBalanced Routing Method Based on Forward-Aware Factor for Wireless Sensor Networks," IEEE Transactions on Industrial Informatics, Vol. 10, No. 1, Pages 766 - 773, February 2014.

14. J. I. Bangash, A. H. Abdullah, M. H. Anisi, and A. W. Khan, "A Sensors, Vol. 14, No. 1, Pages 1322 - 1357, January 2014

15. T. Rault, A. Bouabdallah, and Y. Challal, "Energy Efficiency inWireless Sensor Networks: A Top-Down Survey," Computer Networks, Vol. 67, No. 1, Pages 104 - 122, July 2014.

16. L. Xie, Y. Shi, Y. T. Hou, W. Lou, H. D. Sheral, and S. F. Midkiff, "Multi-Node Wireless Energy Charging in Sensor Networks," IEEE/ACM Transactions on Networking, Vol. 23, No. 2, Pages 437 . 450, April 2015

17. T. K. Jain, D. S. Saini, and S. V. Bhooshan, "Lifetime Optimization of a Multiple Sink Wireless Sensor Network through Energy Balancing," Journal of Sensors, Vol. 2015, Pages 1 - 6, 2015.

18. M. Gholipour, A. T. Haghighat, and M. R. Meybodi, "Hop-by-Hop Traffic-Aware Routing to Congestion Control in Wireless Sensor Networks," EURASIP Journal on Wireless Communications and Networking, Vol. 2015, No. 15, Pages 1 - 13, January 2015. Efficient and Fault Tolerant Routing in Wireless Sensor Networks," Wireless Networks, Vol. 21, No. 1, Pages 251 - 267, January 2015.

20. Quansheng Guan, F. Richard Yu, Shengming Jiang, Victor C. M Leung, Hamid Mehrvar, ?Topology Control in Mobile Ad hoc Networks With Cooperative Communications ?, IEEE Wireless Communications, Page(s): 74 - 79, April 2012. control and routing protocol for multihop wireless sensor networks". protocol in mobile wireless sensor networks. Wireless Survey of Routing Protocols in Wireless Body Sensor Networks,"

19. M. Azharuddin and P. K. Jana, "A Distributed Algorithm for Energy

21. Qian Li, Rose Qingyang Hu, Yi Qian, Geng Wu, ?Cooperative communications for wireless networks: techniques and applications in LTE-advanced systems ?, IEEE Wireless Communications, Volume: 19, Issue: 2, Page(s): 22 - 29, April 2012.

22. W.-H. Chen, A.-C. Pang, A.-C. Pang, and C.-T. F. Chiang, "Crosslayer cooperative routing for vehicular networks," in Proc. IEEE International Computer Symposium (ICS), Dec. 2010, pp. 67 72.

23. R. Madan, N. Mehta, A. Molisch, and J. Zhang, "Energy-efficient decentralized cooperative routing in wireless networks," IEEE Trans. Autom. Control, vol. 54, no. 3, pp. 512-527, Mar. 2009.

24. A. Khandani, J. Abounadi, E. Modiano, and L. Zheng, "Cooperative routing in static wireless networks," IEEE Trans. Commun., vol. 55, no. 11 , pp. $2185-2192$, Nov. 2007.

25. C. Pandana, W. Siriwongpairat, T. Himsoon, and K. Liu, "Distributed cooperative routing algorithms for maximizing network lifetime," in Proc. IEEE Wireless Communications and Networking Conference, (WCNC), Apr. 2006, pp. 451-456.

26. F. Li, K. Wu, and A. Lippman, "Minimum energy cooperative path routing in all-wireless networks: Np-completeness and heuristic algorithms," EURASIP J. Wirel. Commun. Netw., vol. 10, no. 2, pp. 204- 212, June 2008

27. H. Xu, L. Huang, C. Qiao, Y. Zhang, and Q. Sun, "Bandwidth-power aware cooperative multipath routing for wireless multimedia sensor networks," IEEE Trans. Wireless Commun., vol. 11, no. 4, pp. 1532 1543, Apr. 2012

28. S. Chen, M. Huang, Y. Li, Y. Zhu, and Y. Wang, "Energy-balanced cooperative routing in multihop wireless ad hoc networks," in Proc. IEEE International Conference on Communications (ICC), June 2012 pp. 307-311

29. A. Akhtar, M. Nakhai, and A. Aghvami, "Power aware cooperative routing in wireless mesh networks," IEEE Commun. Lett., vol. 16, no. 5, pp. 670-673, May 2012.

30. C. Zhai, J. Liu, L. Zheng, H. Xu, and H. Chen, "Maximise lifetime of wireless sensor networks via a distributed cooperative routing algorithm," Trans. Emerging Telecommun. Technol., vol. 23, no. 5, pp. 414-428, 2012

31. S. Lakshmanan and R. Sivakumar, "Proteus: Multiflow diversity routing for wireless networks with cooperative transmissions," IEEE Trans. Mobile Comput., vol. 12, no. 6, pp. 1146-1159, 2013.

32. Z. Sheng, Z. Ding, and K. Leung, "Distributed and power efficien routing in wireless cooperative networks," in Proc. IEEE International Conference on Communications (ICC), June 2009, pp. 1-5.

33. L. Shi and A. O. Fapojuwo, "Cross-layer optimization with cooperative communication for minimum power cost in packet error rate constrained wireless sensor networks," Ad Hoc Networks, vol 10, no. 7, pp. 1457 - 1468, 2012.

34. Ahmed s. Ibrahim, zhu hany, and k. J. Ray liu, "distributed energyefficient cooperative routing in wireless networks. Ieee transactions on wireless communications, vol. 7, no. 10, october 2008

35. J. Shi, A. Calveras, Y. Cheng and K. Liu, "A Novel Power Efficient Location-Based Cooperative Routing with Transmission PowerUpper-Limit for Wireless Sensor Networks", Sensors 2013, Vol. 13 doi:10.3390/s130506448, pp. 6448-6476.

36. X. Haung, H. Zhai and Y. Fang, "Robust Cooperative Routing Protocol in Mobile Wireless Sensor Networks", IEEE Transaction on Wireless Communications, Vol.7, No.12, pp. 5278-5285, December 2008.

37. F. Akyildiz, W. Su, Y. S. Subramanian and E. Cerci, "A survey on Sensor Networks", IEEE Communication Magazine, Vol.40, issue 8, pp.102-114, August 2002.

38. J. G. Proakis, Digital Communications, McGraw-Hill, 1995.

39. A. Sendonaris and E. Erkip, "User cooperation diversity Part II: Implementation aspects and performance analysis," IEEE Trans. Commun., vol. 51, no. 11, pp. 1939-1948, November 2003.

40. J. N. Laneman, D. N. C Tse, and G. W. Wornell, "Cooperative diversity in wireless networks: Efficient protocols and outage behaviour," IEEE Trans. Inform. Theory, 2004.

41. L. Liu, Z. Wang and M. Zhou, "Energy-Efficient Cooperative Routing for Wireless Sensor Networks Using Space Time Block Code", IEEE International Conference on Systems, Man and Cybernetics, pp.28372842, 7-10 Oct. 2007.

42. L. Liu, Z. Wang and M. Zhou, "Space-Time-Block-Code based Cooperative Routing for WSNs", International Journal of Intelligent Control and Systems, Vol. 14, No. 3, 2009, pp.213-220.

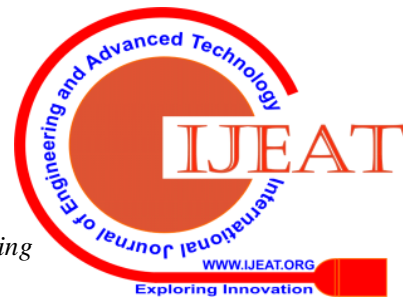




\section{A Research on the Recent Advances in the Development of Energy Efficient Distributed Cooperative Routing Protocol for Wireless Sensor Networks in the Current scenario}

43. H. Takagi, "Queuing Analysis: A foundation of Performance Evaluation, Vacation and Priority systems," Vol. 1, North-Holland, Amsterdam, 1991.

44. L. Cheng, J. Cao, C. Chen, H. Chen, J. Ma and J.I. Siebert, "Cooperative Contention-based forwarding for Wireless Sensor Networks", IWCMC '10, June 28-July 2, 2010, Caen, France.

45. H. Sivsankari, K. Shaila, K.R. Venugopal, S.S. Iyengar and L.M. Panaik, "Energy Efficient Adaptive Cooperative Routing (EEACR) with Multiple Sinks in Wireless Sensor Networks", 7th IEEE Conference on Industrial Electronics and Applications (ICIEA), 2012, pp. 676-681.

46. F. Bouabdallah, N. Bouabdallah and R. Boutaba, "Reliable and Energy Efficient Cooperative Detection in Wireless Sensor Networks", Computer Communications Journal. Elsevier. Vol. 36(5), pp. 520-532, March 2013

47. S.S. Alwakeel, N.A. Al-Nabhan, "A Cooperative Learning Scheme for Energy Efficient Routing in Wireless Sensor Networks," IEEE 11 th International Conference on Machine Learning and Applications (ICMLA), 2012 vol.2, pp.463,468, doi: 10.1109/ICMLA.2012.143.12-15 Dec. 2012.

48. A.N. Beongku and D. T. Trung, "A cooperative routing in mobile adhoc wireless sensor networks with Rayleigh fading environments," International Symposium on Computer Communication Control and Automation (3CA), 2010, vol.1, doi: 10.1109/3CA.2010.5533848, pp.22-25, 5-7 May 2010.

49. L. Bin, W. Wang, Q. Yin, H. Li and H.Wang, "Energy-efficient cooperative geographic routing in wireless sensor networks," IEEE International Conference on Communications (ICC), pp.152-156, 1015 June 2012.

50. R.R. Rout, S.K. Ghosh and S. Chakrabarti, "Co-operative routing for wireless sensor networks using network coding," Wireless Sensor Systems, IET , vol.2, no.2, pp.75-85, doi: 10.1049/iet-wss.2011.0069, June 2012.

51. A. Nieto, and J. Lopez, "Traffic Classifier for Heterogeneous and Cooperative Routing through Wireless Sensor Networks," 26th International Conference on Advanced Information Networking and Applications Workshops (WAINA), 2012, pp.607- 612, doi: 10.1109/WAINA.2012.202,26-29 March 2012.

52. Siyuan Chen, Yang Li , Minsu Huang, Ying Zhu, Yu, "Wang Energy-balanced cooperative routing in multihop wireless networks", Published online: 2 November 2012 _ Springer Science Business Media New York 2012, Wireless Network 2013 19:1087-1099 DOI 10.1007/s1 1276-012-0520-6

53. Ahmed S. Ibrahim, Student Member, IEEE, Zhu Han, Member, IEEE, and K. J. Ray Liu, Fellow, IEEE "Distributed Energy-Efficient Cooperative Routing in Wireless Networks", IEEE transactions on wireless communications, vol. 7, no. 10, October 2008

54. Lu Zhang, Student Member, IEEE, and Leonard J. Cimini, Jr. Fellow, IEEE "Efficient Power Allocation for Decentralized Distributed Space-Time Block Coding", IEEE transactions on wireless communications, vol. 8 , no. 3 , march 2009

55. Mohamed Elhawary and Zygmunt J. Haas, Fellow, IEEE, "Energy Efficient Protocol for Cooperative Networks", IEEE/ACM transactions on networking, vol. 19, no. 2, aril 2011.

56. U.Sandhya, S.Vikram Phaneendra b "Optimization of Energy Usage in Cooperative Networks", International Journal of Computer Trends and Technology (IJCTT) - volume 4 Issue 9- Sep 2013

57. A.F. M. Shahen Shah, Md. Shariful Islam, " A Survey on Cooperative Communication in Wireless Networks" I.J. Intelligent Systems and Applications, 2014, 07, 66-78 Published Online June 2014 in MECS (http://www.mecs-press.org/) DOI 10.5815/ijisa.2014.07.09

58. Ritesh Madan, Member, IEEE, Neelesh B. Mehta, Senior Member, IEEE, Andreas F. Molisch, Fellow, IEEE, and Jin Zhang, Fellow, IEEE, "Energy-Efficient Decentralized Cooperative Routing in Wireless Networks", IEEE transactions on automatic control, vol. 54 no. 3, march 2009

59. Nikolaos A. Pantazis, Stefanos A. Nikolidakis and Dimitrios D Vergados, Senior Member, IEEE Energy-Efficient Routing Protocols in Wireless Sensor Networks: A Survey, IEEE communications surveys \& tutorials, vol. 15, no. 2, second quarter 2013.

60. Charles Pandana, W. Pam Siriwongpairat, Thanongsak Himsoon, and K. J. Ray Liu, "Distributed Cooperative Routing Algorithms for Maximizing Network Lifetime", 1-4244-0270-0/06/ 2006 IEEE.

61. Nikos Dimokas, Dimitrios Katsaros, "Detecting Energy-Efficient Central Nodes for Cooperative Caching in Wireless Sensor Networks", 2013 IEEE 27th International Conference on Advanced Information Networking and Applications 1550-445X/13 2013 IEEE DOI 10.1109/AINA.2013.120
62. S. Manfredi, "Reliable and energy-efficient cooperative routing algorithm for wireless monitoring systems", published in IET Wireless Sensor Systems, Received on 14th March 2011, doi 10.1049/iet-wss.2011.0103.

63. Stefanos A. Nikolidakis, Dionisis Kandris, Dimitrios D. Vergados Christos Douligeris, "Energy Efficient Routing in Wireless Sensor Networks Through Balanced Clustering", Algorithms 2013, 6, 29-42; doi:10.3390/a6010029

64. Lindsey, S.; Raghavendra, C. PEGASIS: Power-Efficient Gathering in Sensor Information Systems. In Proceedings of the IEEE Aerospace Conference, Los Angeles, MT, USA, 2002; pp. 1125 1130.

65. Jung, S, Han, Y, Chung, T, "The Concentric Clustering Scheme for Efficient Energy Consumption in the PEGASIS", In Proceedings of the 9th International Conference on Advanced Communication Technology, Gangwon-Do, Korea, 2007; pp. 260-265.

66. Manjeshwar, A, Agrawal, D. Teen: "A Routing Protocol for Enhanced Efficiency in Wireless Sensor Networks", In Proceedings of the 15th International Parallel and Distributed Processing Symposium (IPDPS'01) Workshops, San Francisco, CA, USA, 2001; pp. 2009 2015.

67. Yang, Y.; Wu, H.; Chen, H. SHORT: Shortest Hop Routing Tree for Wireless Sensor Networks. In Proceedings of the IEEE International Conference on Communications, Istanbul, Turkey, 2006; pp. 34503454.

68. Lotf, J.; Bonab, M.; Khorsandi, S. A Novel Cluster-based Routing Protocol with Extending Lifetime for Wireless Sensor Networks. In Proceedings of the 5th International Conference on Wireless and Optical Communications Networks, Surabaya, India, 2008; pp. 1-5.

69. Allirani, A.; Suganthi, M. An Energy Efficient Cluster Formation Protocol with Low Latency In Wireless Sensor Networks. World Acad. Sci., Eng. Tech. 2009, 51, 1-7.

70. Muruganathan, S.; Ma, D.; Bhasin, R.; Fapojuwo, A. A Centralized Energy-Efficient Routing Protocol for Wireless Sensor Networks. IEEE Radio Commun. 2005, 43, 8-13. 